\title{
Erratum to: ExCAPE-DB: an integrated large scale dataset facilitating Big Data analysis in chemogenomics
}

Jiangming Sun ${ }^{1 *}$, Nina Jeliazkova ${ }^{2}$, Vladimir Chupakhin ${ }^{3}$, Jose-Felipe Golib-Dzib ${ }^{4}$, Ola Engkvist ${ }^{1}$, Lars Carlsson ${ }^{1}$, Jörg Wegner ${ }^{3}$, Hugo Ceulemans ${ }^{3}$, Ivan Georgiev², Vedrin Jeliazkov², Nikolay Kochev ${ }^{2,5}$, Thomas J. Ashby ${ }^{6}$ and Hongming Chen ${ }^{1 *}$

\section{Erratum to: J Cheminform (2017) 9:17 DOI 10.1186/s13321-017-0203-5}

After publication of this work [1], it was noticed that the author's name was spelt incorrectly. His name should be spelt as: Vladimir Chupakhin. The ' $h$ ' was missing from his surname.

The publisher apologises for these errors.

The original article has been updated.

\begin{abstract}
Author details
${ }^{1}$ Discovery Sciences, Innovative Medicines and Early Development Biotech Unit, AstraZeneca R\&D Gothenburg, 43183 Mölndal, Sweden. ${ }^{2}$ Ideaconsult Ltd., 4. Angel Kanchev Str., 1000 Sofia, Bulgaria. ${ }^{3}$ Computational Biology, Discovery Sciences, Janssen Pharmaceutica NV, Turnhoutseweg 30, 2349 Beerse, Belgium. ${ }^{4}$ Computational Biology, Discovery Sciences, Janssen Cilag SA, Calle Río Jarama, 71A, 45007 Toledo, Spain. ${ }^{5}$ Department of Analytical Chemistry and Computer Chemistry, University of Plovdiv, Plovdiv, Bulgaria. ${ }^{6}$ Imec vzw, Kappeldreef 75, 3001 Louvain, Belgium.
\end{abstract}

The online version of the original article can be found under doi:10.1186/s13321-017-0203-5.

\section{Publisher's Note}

Springer Nature remains neutral with regard to jurisdictional claims in published maps and institutional affiliations.

Received: 24 May 2017 Accepted: 24 May 2017

Published online: 14 June 2017

\section{Reference}

1. Sun J, Jeliazkova N, Chupakhin V, Golib-Dzib J-F, Engkvist O, Carlsson L et al (2017) ExCAPE-DB: an integrated large scale dataset facilitating Big Data analysis in chemogenomics. J Cheminform 9:17

\footnotetext{
*Correspondence: Jiangming.Sun@astrazeneca.com;

hongming.chen@astrazeneca.com

1 Discovery Sciences, Innovative Medicines and Early Development

Biotech Unit, AstraZeneca R\&D Gothenburg, 43183 Mölndal, Sweden
} (http://creativecommons.org/licenses/by/4.0/), which permits unrestricted use, distribution, and reproduction in any medium, provided you give appropriate credit to the original author(s) and the source, provide a link to the Creative Commons license, and indicate if changes were made. 